\title{
Molecular Switches as Platforms for Information Processing
}

\author{
Uwe Pischel* \\ Grammaticakis-Neumann Award 2013
}

\begin{abstract}
This article shows how to teach molecules the basics of logic and how to integrate complicated logic operations with relatively simple molecules. A special focus is put on the realization of multi-level fluorescent switches with chemical inputs as well as on the demonstration of various memory-based operations with alloptical photochromic switches.
\end{abstract}

Keywords: Chemosensors · Fluorescence $\cdot$ Logic $\cdot$ Photochromism · Switches

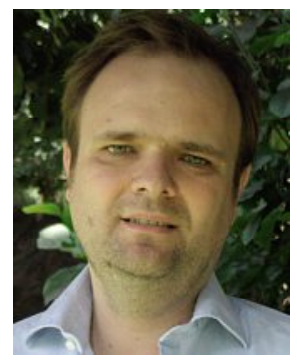

Uwe Pischel, born 1973 in Germany, studied chemistry at the Technical University Dresden and the Humboldt University Berlin. In 2001 he obtained his $\mathrm{PhD}$ under the guidance of Prof. Werner M. Nau at the University of Basel. After a short postdoctoral stay at the Technical University of Valencia he held several positions as Junior Group Leader at the University of Porto and the Technical University of Valencia. Since 2007 he leads the photochemistry group at the University of Huelva in Spain and is currently Profesor Titular in Organic Chemistry. He is recipient of a Ramón y Cajal Fellowship (2006-2009), the Award of the Faculty of Philosophy and Natural Sciences of the University of Basel (2002), the Albert-Weller Prize of the Photochemistry Division of the German Chemical Society and Bunsen Society (2003), and the Grammaticakis-Neumann Award (2013).
${ }^{*}$ Correspondence: Dr. U. Pischel

University of Huelva

Center for Research in Sustainable Chemistry and

Department of Chemical Engineering, Physical

Chemistry, and Organic Chemistry

Campus de El Carmen, $\mathrm{s} / \mathrm{n}$

E-21071 Huelva, Spain

Tel.: +34959219982

E-mail: uwe.pischel@diq.uhu.es

\section{Introduction}

Logic decisions mark our daily life, be it either consciously or unconsciously. For example, simple "if... then..." constructions such as "if the sun shines today, then it will be warm" are likewise described by a logic operation called IMPLICATION. ${ }^{1]}$ Information technologies based on computing pace the rhythm of modern societies and social relationships. At the center of these developments are logic decisions that are based on ones and zeros; the binary code. However, while computer chips work with electrical input and output signals of silicon-based semiconductor logic gates and circuits, in principle any other form of signals and processing units can be imagined. This lies in the universality of the concept of logic. Hence, it is not surprising that the idea of using molecules for information processing has found more and more followers since the works by Aviram ${ }^{[2]}$ and later on the first report of a molecular logic AND gate by the de Silva group. ${ }^{[3]}$ Fluorescent compounds have been a preferential choice for implementing molecular logic devices since the early days of this field. ${ }^{[4-6]}$ This has several reasons, one of which is the ease of design based on the control of well-known excited-state processes such as photoinduced electron transfer (PeT) or energy transfer (EnT). Consequently, the field has always had tight connections with fluorescent chemosensors. ${ }^{[7]}$ From a simplified and formal point of view a chemosensor is a molecular logic gate. Taking the example of a fluorescent off-on chemosensor the following logic connotation applies: if no chemical analyte (input) is present the fluorescence signal (output) is low or even totally absent; if the chemical analyte is present and above a certain concentration threshold the fluorescence signal is high (see examples in Fig. 1) ${ }^{[8,9]}$ If "low (or absent)" and "high" are translated into the binary numbers 0 and 1 , respectively, then the logic YES or IDENTITY operation is straightforward identified: input $=0$ leads to output $=0$ and input $=1$ leads to output $=1$. This is a trivial case which, however,

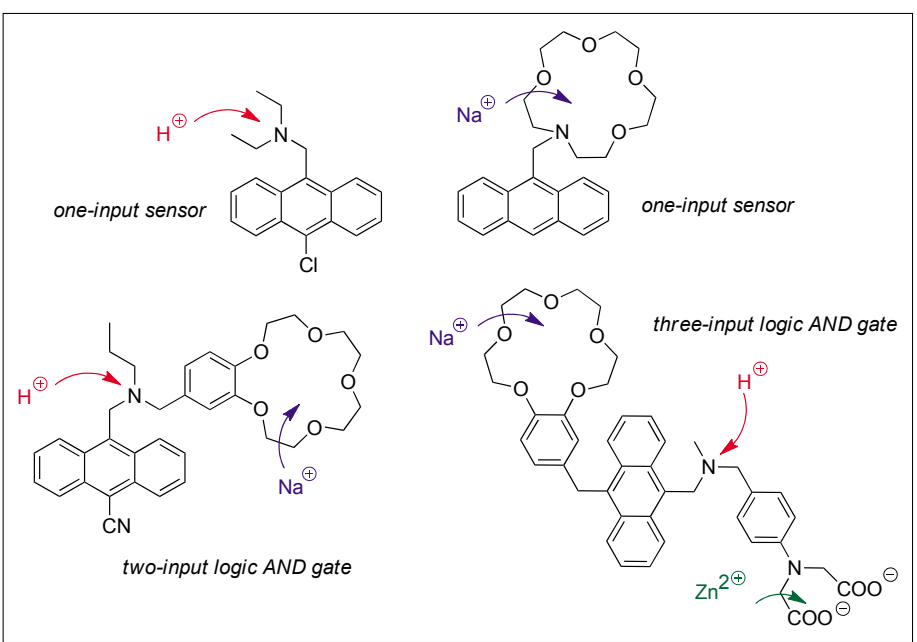

Fig. 1. One-, two-, and three-input systems based on anthracene fluorescence output. 
can be expanded to a higher complexity by combining two or more inputs that are processed into a single output. Examples for such combinatorial chemosensors are de Silva's two-input and three-input AND gates (see Fig. 1), ${ }^{[3,10]}$ where all chemical inputs (i.e. analytes) must be detected by the respective receptors in order to observe a high fluorescence signal. Sometimes it is not so much the specific detection of single analytes that interests, but simply the detection of a combinatorial situation (for example AND) of several analytes. Here is a daily life example that all of us have already experienced: when medical doctors read a blood analysis they rate the measured data (inputs) against a reference value and then combine the binary results (values above or below reference) logically (e.g. AND, OR, etc.) to reach a first diagnosis (output).

With these few considerations it should be clear that the application of logic in molecular sciences can provide interesting approaches to analytical chemistry, especially in the context of clinical diagnostics. This has been developed further and beyond the context of the fluorescent chemosensing of simple ionic analytes. An instructive example is the AND fluorescence detection of bacterial DNA and a pro-inflammatory cytokine as combinatorial biomarkers of infections of the respiratory apparatus. ${ }^{[11]}$ In other works, diagnostic properties were combined with actuation in form of therapy, e.g. the release of a drug or activation of a pro-drug. Singlet oxygen is used as agent in photodynamic therapy of tumors. In this context, the Akkaya group has reported an interesting example of photosensitized singlet oxygen production by using a Bodipy dye that depends on the simultaneous fulfillment of tumor-specific analytical parameters such as sodium ion concentration and $\mathrm{pH}$. In the specific case this translates into an AND logic operation. ${ }^{[12]}$ In a follow-up system the $\mathrm{pH}$-dependent singlet oxygen production was combined with the fluorescence output of a Bodipy dye, leading to a theranostic (therapeutic and diagnostic) device that resembles the logic behavior of a 1:2 demultiplexer (DEMUX). ${ }^{[13]}$ Another work that is related to the activation of a doxorubicin pro-drug through the logically linked biocatalytic action of one OR another enzyme was published by the Shabat group. ${ }^{[14]}$ Finally, the $\mathrm{pH}$ - and light-dependent release of a model drug from the pores of a mechanized nanoparticle serves here to highlight the combination of molecular logic with materials chemistry. ${ }^{[15]}$

Leaving the relation of molecular logic and analytical applications aside, the approach has another very obvious connotation: that of building a molecular computer in a bottom-up approach. Currently, semi- conductor industry is struggling to keep up with Moore's law that predicts that the number of transistors in a circuit doubles every two years. ${ }^{[16]}$ The technological problems that are inherent to the top-down fabrication of semiconductor chips constitute a bottleneck for the chip industry. An alternative approach is to "teach" molecules to assume the functions of conventional logic gates, transistors, etc. and to assemble molecular computers with a higher density of functional information processing units. Naturally, this is an enormous task bearing many problems. As will be shown in this account, unimolecular systems can mimic very complicated logic circuits that would require many wired single logic gates in silicon-based circuitry. It can be also imagined that these functional units can be assembled into extended supramolecular arrays. However, one of the most complex challenges is to make these functional units communicate with each other. This is the problem of concatenation. In the so far mentioned systems chemical inputs were processed into an optical output (fluorescence). This inhomogeneity of the signals impedes direct gate-to-gate communication. In order to overcome this problem several strategies were published as proofof-principle, among them communication via EnT[17] or through the generation of chemical species such as protons ${ }^{[18]}$ or singlet oxygen ${ }^{[19]}$ as output signals.

All-optical logic switches, i.e. those that use exclusively optical inputs and outputs, are a promising alternative to approach the concatenation problem. The inherent homogeneity of the involved signals, the often clean and resettable (reversible) photochemical reactions, and the possibility to deliver inputs and read outputs remotely make photochromic switches interesting platforms for molecular logic operations. ${ }^{20-22]}$ (Multi)photochromic systems and their use for molecular logic will play a central role in this account. However, first some molecular logic switches that process chemical inputs and provide fluorescence changes as outputs will be discussed. The following systems are presented with a strong focus on our own work.

\section{Molecular Switches with Chemical Inputs: Systems for Multi-level Logic}

As discussed in the Introduction, fluorescent systems which change their optical properties upon recognition of chemical input signals can be straightforwardly interpreted as chemosensors. For these purposes it is sufficient to define two signal levels (low and high) according to binary coding. However, for information process- ing with a view on molecular computing it is desirable to develop switches that can differentiate more than two levels for the input and/or output signals. Such multivalued switches, e.g. ternary and quaternary switches, are challenging from the point of view of the molecular design.

The first system that will be presented here is compound $\mathbf{1}$ which is composed of two electronically different naphthalimide chromophores (Fig. 2).[23] They are linked by an amino-containing spacer. The aromatic substitution with an electron-donating amino group at the 4 position of one of the chromophores confers internal-chargetransfer (ICT) character to this moiety (ANI). This leads to a significant red-shift of the absorption and emission spectra with respect to the parent chromophore (NI). Upon selective excitation of the NI chromophore an efficient EnT process to the ANI moiety applies. In accordance, the Förster theory of resonance energy transfer predicts a critical radius $R_{0}$ of $19 \AA$, well above the real distance between the two chromophores. Consequently, exclusively the green fluorescence of ANI is observed. However, the EnT process is in competition with an exergonic PeT from the electrondonating amino group of the spacer. As a special twist this PeT is only operative with the excited NI chromophore, but impossible with the excited state ANI moiety. ${ }^{[24]}$ The competition between EnT and PeT for the excited state NI can be suppressed by protonation of the spacer amino group, thereby converting it into a redox-inactive trialkylammonium ion. Starting from this protonated form, which shows the highest ANI fluorescence signal upon excitation of NI, several consecutive processes are possible upon addition of fluoride ions $\left(\mathrm{F}^{-}\right)$that act as strong base in organic solvents. In a first step ( $\mu \mathrm{M}$ addition of $\left.\mathrm{F}^{-}\right)$, the ammonium ion is deprotonated, thereby re-activating the PeT (see Fig. 2). This is accompanied by $c a$. $41 \%$ reduction of the fluorescence signal. Upon addition of higher $\mathrm{F}^{-}$concentrations ( $\mathrm{mM}$ range) a second deprotonation at the amino group of the ANI moiety is observed, leading to quantitative fluorescence quenching. Expressing the fluoride concentration as $\mathrm{pF}=-\log \left[\mathrm{F}^{-}\right]$, three input levels are defined and which can be assigned ternary values of 0,1 , and 2 . Also the fluorescence output signal exists in three discrete states that are defined as high (2), medium (1), and low (0) (Fig. 2). By combining this information in a truth table, a ternary NOR logic gate is obtained. While multi-valued switching was discussed before this work, ${ }^{[25-27]}$ this is the first functional assignment of a ternary logic gate.

Compound 2 (Fig. 3) features two proton receptors, one piperazinyl and one quinolinyl moiety, with well differenti- 


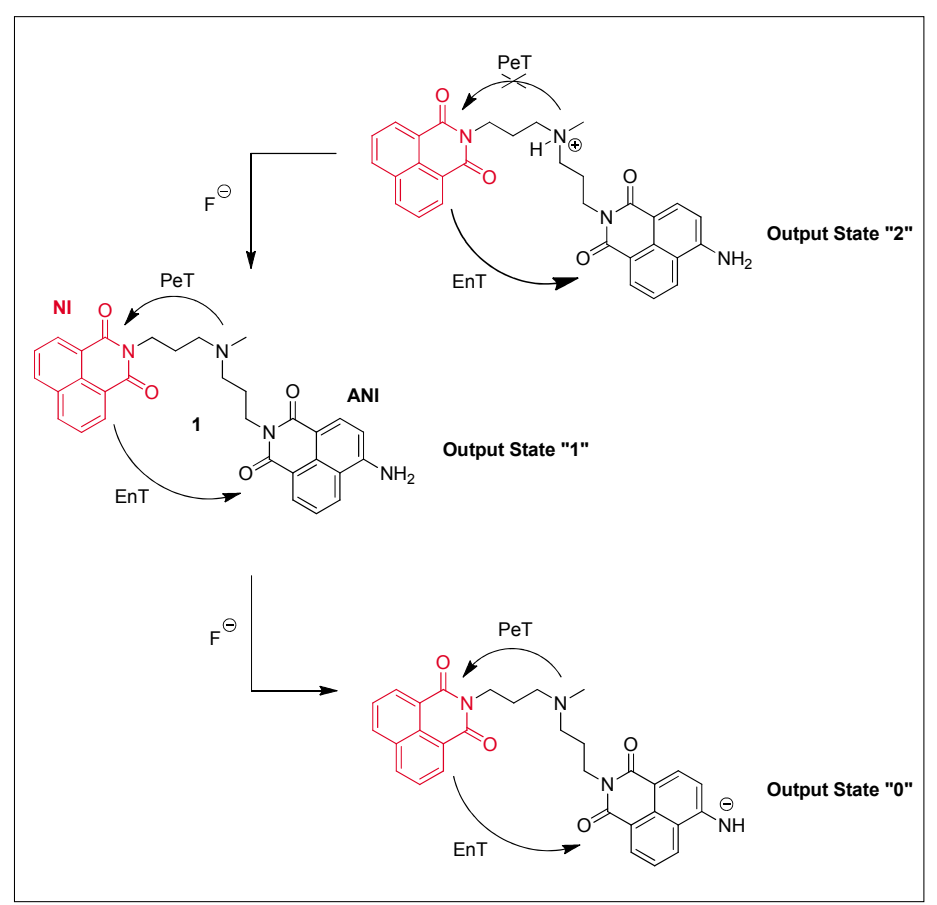

Fig. 2. Ternary molecular logic switching based on compound 1. ated protonation constants. ${ }^{[28]}$ This enables their sequential protonation. Both receptors are connected to a central aminonaphthalimide fluorophore. In the unprotonated state the conjugate shows low fluorescence $\left(\Phi_{\mathrm{f}}=0.017\right)$ due to an efficient PeT from the electron-donating piperazinyl moiety to the electron-accepting fluorophore. Upon protonation with one equivalent of acid at the lateral piperazinyl nitrogen this quenching pathway is deactivated, which is accompanied by a dramatic fluorescence increase $\left(\Phi_{\mathrm{f}}=0.67\right)$. However, upon a second protonation (this time at the quinoline) again a strong quenching $\left(\Phi_{\mathrm{f}}=0.017\right)$, presumably due to hydrogen-bonding interactions between the formed quinolinium ion and the imide carbonyl groups, is observed. Hence, upon application of three proton input levels, assigned as 0,1 , and 2 , an off-on-off fluorescence switching is obtained. This switch resembles the operation of a T-latch (toggle latch). A one-time application of protons produces a 1 output (high fluorescence) while a second application sets the system back to a 0 output (low fluorescence). A well-known analogy of a T-latch is a conventional light switch. The system can be reset by addition of a base and a stable performance over several cycles is obtained. However, waste products such as water and salts are formed in each neutralization step. This is a general problem for logic switches with chemical inputs.

In a more recent work we aimed at the implementation of proton-induced multilevel logic switching with a novel class of fluorophores that is based on borylated arylisoquinolines. We coined the term BAI dyes for these compounds; see some structures in Fig. 4. These dyes show an inter- esting photophysical behavior that derives from the ICT properties of the emissive excited state.[29-31] The ICT characteristics can be conveniently fine-tuned by changing substitution patterns. Compounds 3-6 shown in Fig. 4 were designed to enable stepwise protonation at lateral amino functions and at the isoquinolinyl moiety.[29] In general, similar off-on-off behavior as seen for compound $\mathbf{2}$ could be expected and indeed, for compound $\mathbf{3}$ this is the case. The first protonation step at the piperazinyl moiety blocks PeT, as discussed for $\mathbf{2}$, and the second protonation at the isoquinolinyl leads to quenching. However, depending on the redox properties of the aryl moiety (phenyl, naphthyl or anthryl) slightly different observations are made for the other dyes. For example dye $\mathbf{4}$ behaves as switch with a ternary medium-on-off fluorescence output pattern. On the other hand, dye 5 has three available protonation sites, and thus, a quaternary switching behavior applies. ${ }^{[32]}$ Finally, dye $\mathbf{6}$ behaves as simple on-off switch.

\section{Photochromic Systems for Information Processing}

Photochromic switches have been exploited abundantly for example for the light-induced control of bioimaging, ${ }^{[33]}$ biological functions, ${ }^{[34]}$ and the encoding of light signals. ${ }^{[35]}$ Another field of application is their use for information storage. ${ }^{[36-39]}$ The reversible photoswitching between

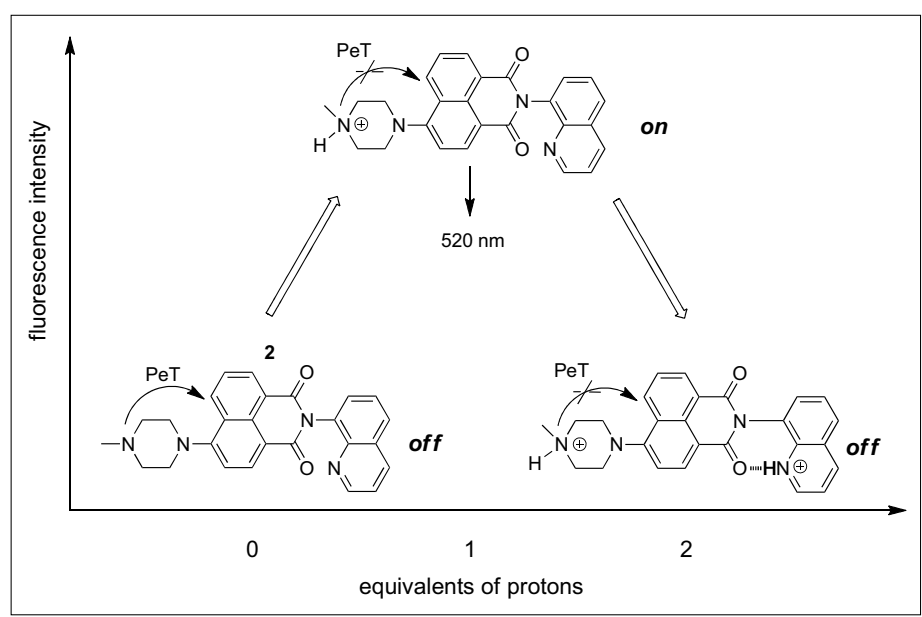

Fig. 3. Off-on-off fluorescence switching upon sequential protonation of compound 2.

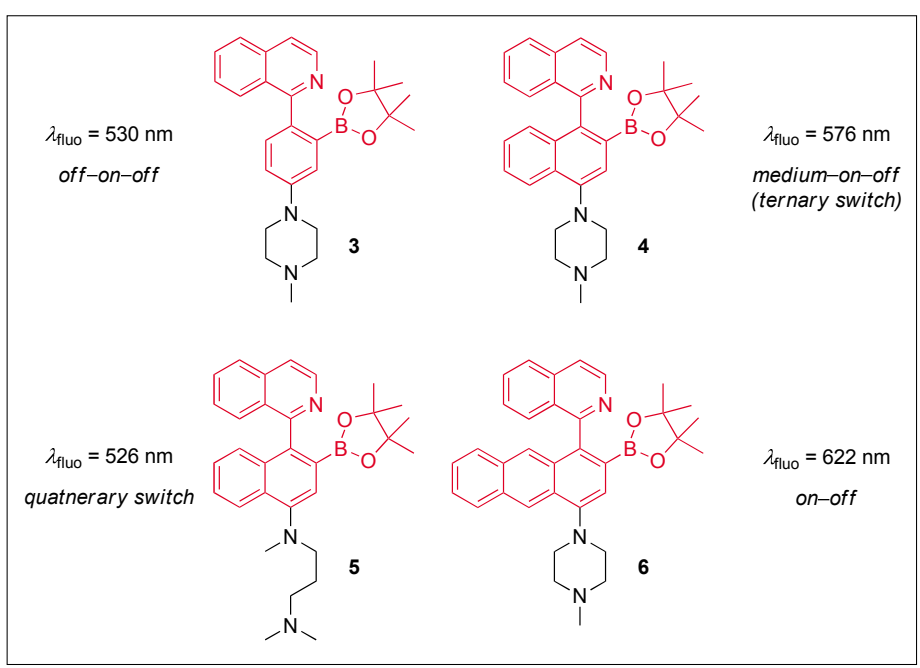

Fig. 4. Structures of fluorescent borylated arylisoquinolines (BAI dyes) 3-6 for multilevel switching. 
two forms of a photochromic compound can be interpreted as writing and erasing of information. The photochromic transformation leads to pronounced changes of absorption spectra and sometimes also of fluorescence properties, such that an intelligent choice of the optical output channel provides a straightforward means to define 0 and 1 states. On the one hand, such photochromic molecular memories are simple examples for the storage of information. On the other hand, the processing of information, provided in form of excitation light of certain wavelengths, has received also much attention in recent years. ${ }^{[20-22]}$ In the following few examples of our research that has been undertaken in the latter context will be described.

In this account dithienylethenes, fulgimides, and spiropyrans play a prominent role. ${ }^{[40]}$ Spiropyrans can be transformed between three forms: the ring-closed colorless spiro form (SP), the ring-opened colored merocyanine form (ME), and the protonated merocyanine form (MEH) (Fig. 5). Irradiation of SP with UV light yields $\mathrm{ME}$, that be converted back to SP either by irradiation with visible light or in a thermally activated process. The addition of acid to ME produces the thermally stable MEH form with a blue-shifted absorption spectrum. Application of visible light of appropriate wavelength triggers ring closure back to SP and the release of a proton. The compounds 7-9 are spiropyrans that are linked to a fluorophore moiety, i.e. dansyl, aminonaphthalimidyl or perylenyl (see structures in Fig. 6). ${ }^{[41]}$ These fluorophores were chosen to fulfill the following design criteria: a) a significant spectral overlap of the fluorophore emission spectrum with the absorption spectra of the ME and $\mathrm{MEH}$ forms of the spiropyran and $\mathrm{b}$ ) a negligible spectral overlap in the case of the SP form. Hence, it could be expected that fluorescence quenching by EnT is pronounced with both ring-opened forms, $\mathrm{ME}$ and MEH, but not with the SP form. This designed peculiarity can be used for the logic operation of a molecular keypad lock (Fig. 7) where not only the "right" two inputs have to be applied, but also in the "right" order. ${ }^{[42,43]}$ A corresponding example of everyday life is the operation of an automatic teller machine. In the discussed molecular example only two inputs $\left(I_{1}\right.$ and $\left.I_{2}\right)$ are used, which constitutes a proof-of-principle. The system is set to the $\mathrm{MEH}$ form as initial state. The input $I_{1}$ is defined as addition of base which triggers the $\mathrm{MEH} \rightarrow \mathrm{ME}$ transformation. The input $I_{2}$ is irradiation with green light that converts ME to SP, but not MEH which lacks absorption in this spectral region. Hence, if first $I_{1}$ and then $I_{2}$ are applied, the following sequence results: $\mathrm{MEH} \rightarrow \mathrm{ME} \rightarrow \mathrm{SP}$. Consequently, a strong emission signal of the fluorophore is observable in this case (binary 1) because energy transfer quenching is not operative in the SP form of the dyads. However, if first green-light irradiation (input $I_{2}$ ) and then base addition (input $I_{1}$ ) is chosen as the sequence, then a simple $\mathrm{MEH} \rightarrow \mathrm{ME}$ transformation is the result and fluorescence quenching in the $\mathrm{ME}$ form keeps the output emission signal low (binary 0). This simple example, where

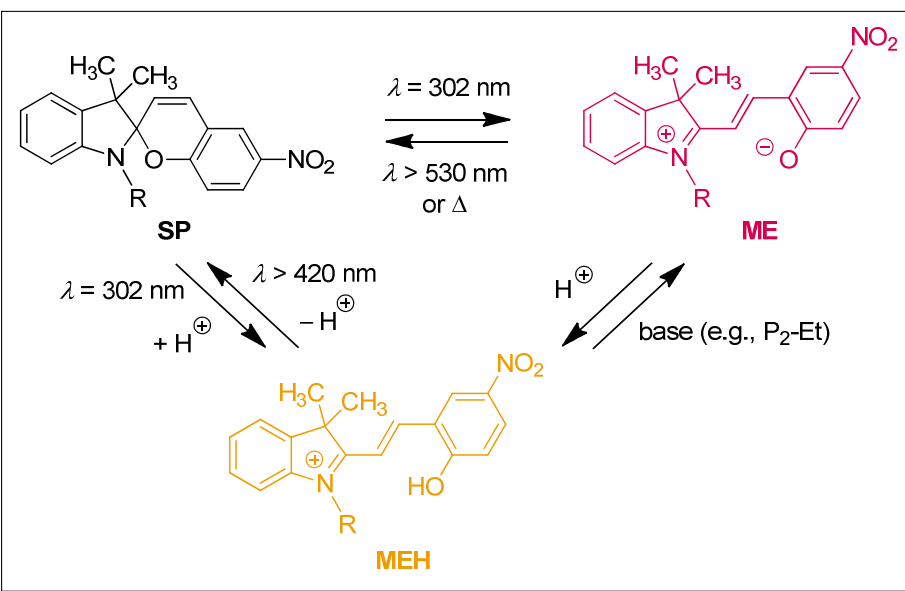

Fig. 5. Photochemical and chemical switching between open and closed spiropyran forms.
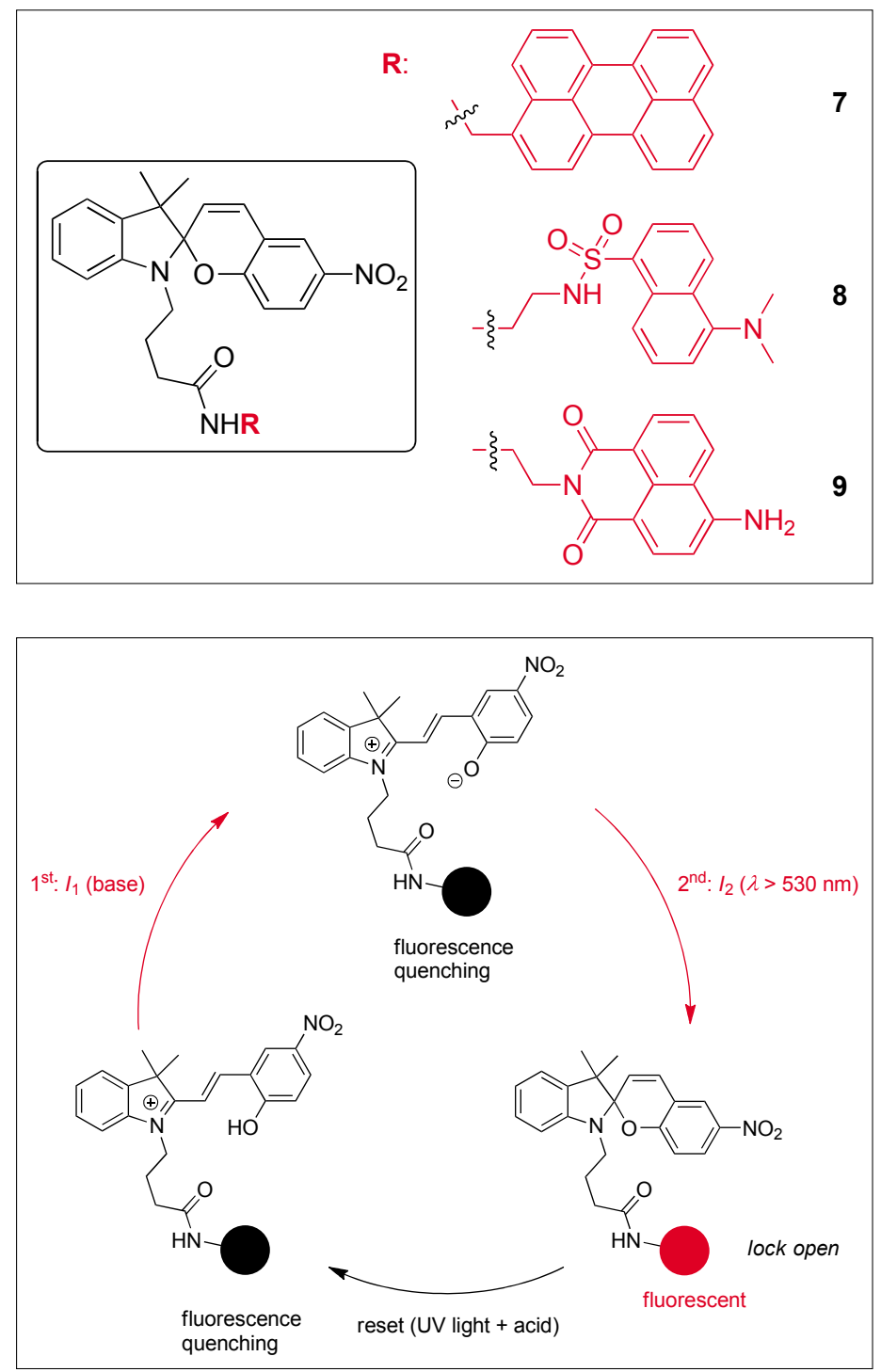

Fig. 6. Spiropyrans 7-9 with appended fluorophores for photochromic switching of energy transfer.

Fig. 7. Illustration of the sequential input application for the activation of a molecular keypad lock based on the compounds 7-9. 
Fig. 8. The device can be switched between two $Q$ states: $Q=0$ and $Q=1$. A change of the $Q$ state from 0 to 1 is observed for the activation of the Set input $S$. The opposite switching from 1 to 0 is obtained for the application of the Reset input $(R=1)$. The $Q$ state remains unchanged for the "do nothing" situation $(S=R=0)$ or the further application of $S=1$ or $R=1$ over the $Q$ $=1$ or $Q=0$ state, respectively. Finally, the simultaneous activation of $R$ and $S$ ( $S$ $=R=1$ ) is physically meaningless. From these statements the truth table shown in Fig. 8 is derived, with $R$ and $S$ as inputs and the corresponding assignments of the current and next $Q$ state, $Q_{\text {current }}$ and $Q_{\text {next' }}$, respectively. Exactly this behavior can be implemented at the molecular level with the dithienylethene 10. ${ }^{[44]}$ Starting from the colorless open form 100 the colored closed form 10c $\left(\lambda_{\text {max }}=595 \mathrm{~nm}\right)$ can be obtained by UV-light irradiation $(\lambda=302$ $\mathrm{nm})$. The reading of the absorbance at 595 nm defines the $Q$ state as 0 or 1 , while the UV light is the $S$ input. The $R$ input is broadband visible light with $\lambda>450 \mathrm{~nm}$, which induces the ring-opening reaction of the dithienylethene. The suggestion to use photochromic systems for the demonstration of photo-addressable memories goes back to the pioneering work of Hirshberg, who proposed the above-discussed spiropyrans for this purpose. ${ }^{[45]}$ Recent work employed also chemically switchable redox systems as S/R-latches. ${ }^{[46,47]}$

Another latch function that finds wide application in conventional computing is the D-latch (delay latch). This device has two inputs, a clock input $(C)$ and a data input $(I)$. The output state is again denominated as $Q$, differentiated by $Q_{\text {current }}$ and $Q_{\text {next }}$. Whenever $C=0$ then $Q_{\text {current }}$ and $Q_{\text {next }}$ have the same binary value. However, when $C=1$ then $Q_{\text {next }}$ has the same value as the data input $I$. This logic function was implemented with the fulgimide derivate 11 (see structure in Fig. 9). [48]

The fulgimide exists as $E$ or $Z$ configuration in its open form $(11 \mathrm{oE}$ or $11 \mathrm{oZ})$, both of which are photo-equilibrated by UV light irradiation. The closed colored form $\left(\lambda_{\max }=523 \mathrm{~nm}\right) 11 \mathrm{c}$ is formed from $11 \mathrm{oE}$ in a $6 \pi$ electrocyclic reaction that is triggered by UV light. Irradiation of 11c with visible light generates back the $11 \mathrm{oE}$ form. These processes are summarized in Fig. 9. Interestingly, the closed form shows a weak, but measurable fluorescence signal with a maximum around $640 \mathrm{~nm}$. This is chosen as output signal which defines the $Q$ state of the device: a fluorescence signal above the threshold means 1 , while any other situation corresponds to 0 . As inputs irradiation at $532 \mathrm{~nm}$ and $1064 \mathrm{~nm}$ with a NdYAG laser was chosen for the $C$ and $I$ input, respectively. Leaving the clock input inactivated $(C=0)$ means only 1064

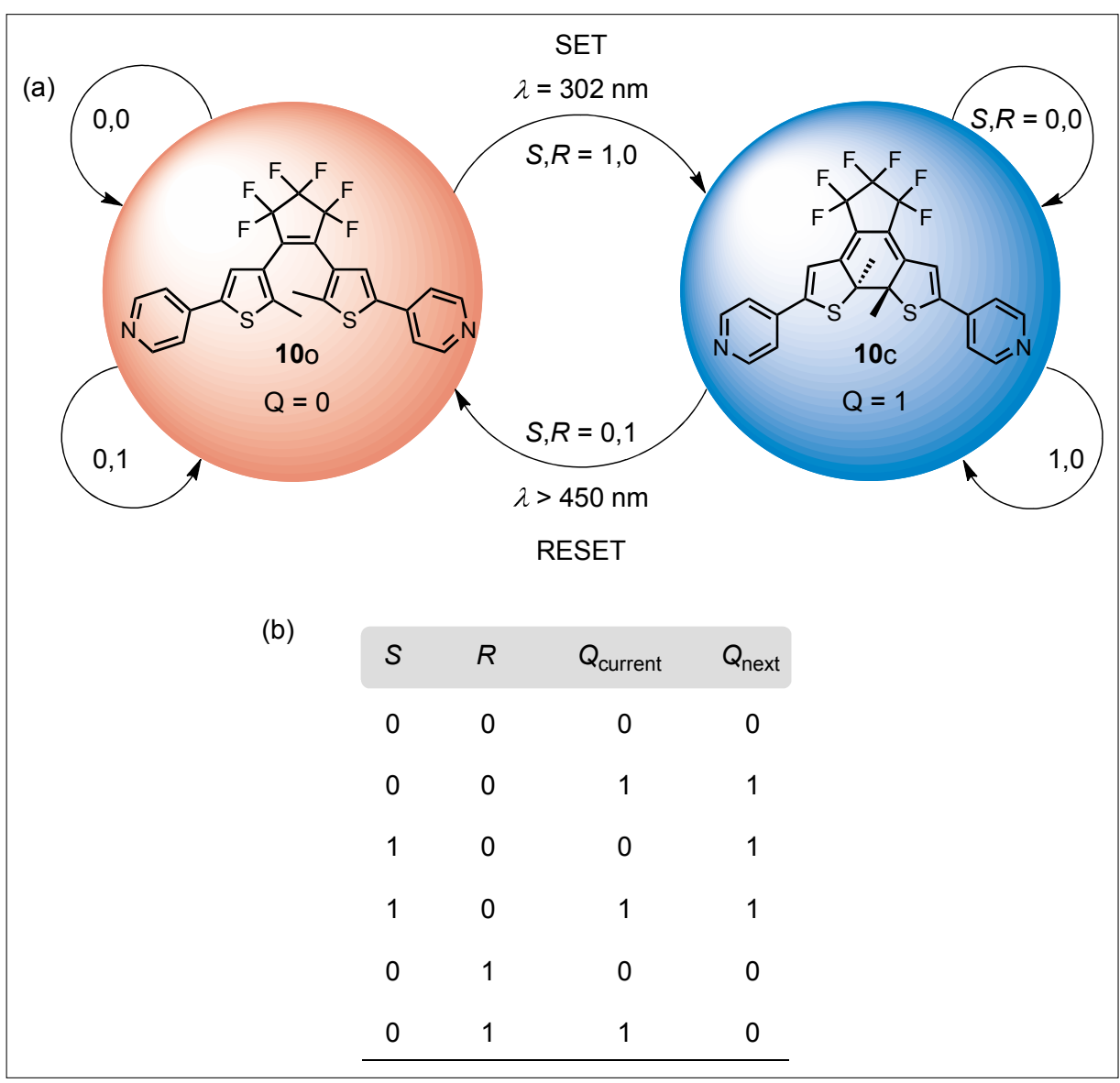

Fig. 8. a) Schematic illustration of the function of an S/R-latch with the example of the dithienylethene 10. b) Truth table for an S/R-latch.

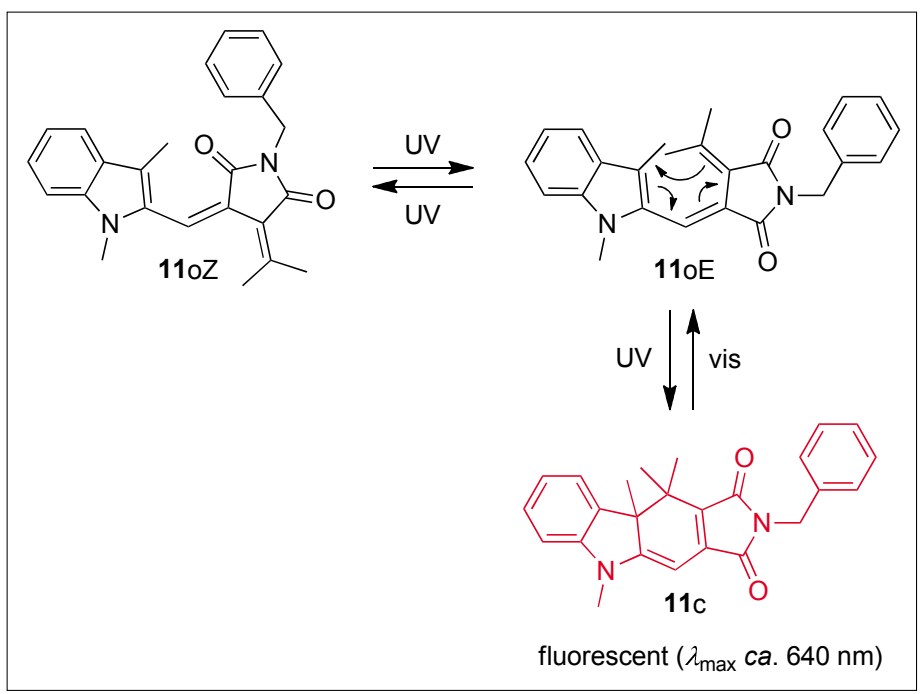

Fig. 9. Photochemical transformations of the fulgimide 11.

$\mathrm{nm}$ IR light $(I=1)$ is applied or nothing is done to the system $(C=I=0)$. All forms of $\mathbf{1 1}$ are thermally stable and hence, no changes are observed comparing $Q_{\text {current }}$ and $Q_{\text {next }}$. In other words the system remains in its current state. However, using visible light in form of irradiation at 532 $\mathrm{nm}(\mathrm{C}=1)$ changes the scenario. On the one hand, for $C=1$ and $I=0$ the system is converted to the non-fluorescent $11 \mathrm{oE}$ or remains in this form $\left(Q_{\text {next }}=0\right)$. On the other hand, by combining $532 \mathrm{~nm}$ and 1064 $\mathrm{nm}$ light in a third-harmonic-generating crystal ( $C=1$ and $I=1) 355 \mathrm{~nm}$ UV light is generated and the photochromic system is transformed into the closed fluorescent form 11c or remains in this form $\left(Q_{\text {next }}=1\right)$. These observations correspond to the described function of a D-latch. The switching between the open and closed forms of 11 can be repeated at least ten times without loss of performance.

Up to now single types of photochromic systems were discussed in this account. However, by combination of several photochromic switches in a uni- 
molecular system and by assuring their selective excitation and independent transformations the combinatorial possibilities of input as well as output signals can be significantly extended. This idea has been put into practice with the triad 12 (see structure in Fig. 10), which integrates fulgimide and dithienylethene photochromic switches in one compound. ${ }^{49]}$ In total six isomeric configurations are possible out of which only the ones that contain the identical fulgimides in the same form (open (o) or closed (c)) are functionally relevant. The resulting four isomers are denominated as $12 \mathrm{o} / \mathrm{o}, 12 \mathrm{o} / \mathrm{c}, 12 \mathrm{c} / \mathrm{o}$, and $12 \mathrm{c} / \mathrm{c}$ (with 12FG/DTE). The network that results from the transformations by using light of different excitation wavelengths (302 nm, $366 \mathrm{~nm}, 397 \mathrm{~nm}$, green light, and red light) is shown in Fig. 10. By choosing appropriate output channels (absorbances $A$ at $393 \mathrm{~nm}, 475 \mathrm{~nm}, 535 \mathrm{~nm}, 625 \mathrm{~nm}$, $|\triangle A|$ at $393 \mathrm{~nm}$, and emission at $624 \mathrm{~nm}$ ) up to twelve different logic operations can be functionally integrated into a single system. Among them are simple logic gates (AND, XOR, INHIBIT, TRANSFER) and advanced functions (half-adder, half-subtractor, 4:2 encoder, 2:4 decoder, 2:1 multiplexer, 1:2 demultiplexer, molecular keypad lock, logically reversible switches). With a slightly different set of inputs also a parity generator and parity checker were mimicked. ${ }^{[50]}$ Importantly, all functions are based on an all-optical operation and can be cleanly reset by re-creation of the initial state 120 /o by irradiation with green light.

To illustrate the function of triad $\mathbf{1 2}$ two selected logic operations will be briefly described here: the molecular keypad lock and the logically reversible operation. The molecular keypad function employs 366 $\mathrm{nm}$ UV light and red light as the inputs $I_{1}$ and $I_{2}$, respectively (see Fig. 11). Again, as discussed above for the D-latch, the fluorescence of the closed fulgimide $\left(\lambda_{\max }=\right.$ $624 \mathrm{~nm})$ is used as output signal. Starting from the all-open form, 12o/o, irradiation at $366 \mathrm{~nm}$ isomerizes both photochromic switches, resulting in the isomer $12 \mathrm{c} / \mathrm{c}$. Subsequent irradiation with red light selectively isomerizes the ring-closed form of the dithienylethene, yielding 12c/o (see structure in Fig. 10). This isomer is fluorescent, while in the isomer $12 \mathrm{c} / \mathrm{c}$ an efficient Förster resonance energy transfer $\left(R_{0}\right.$ $=23 \AA$ ) quenches the fulgimide fluorescence. ${ }^{[51]}$ By changing the order of excitation light application (red light before 366 $\mathrm{nm}$ UV light) the non-fluorescent $12 \mathrm{c} / \mathrm{c}$ is formed. Hence, only the sequence " $I_{1}$ before $I_{2}$ " enables the observation of fulgimide fluorescence (output $=1$ signalizing the open lock), while the reverse input order leaves the lock closed (output $=0$ ).

A second example is the implementation of a logically reversible logic opera-

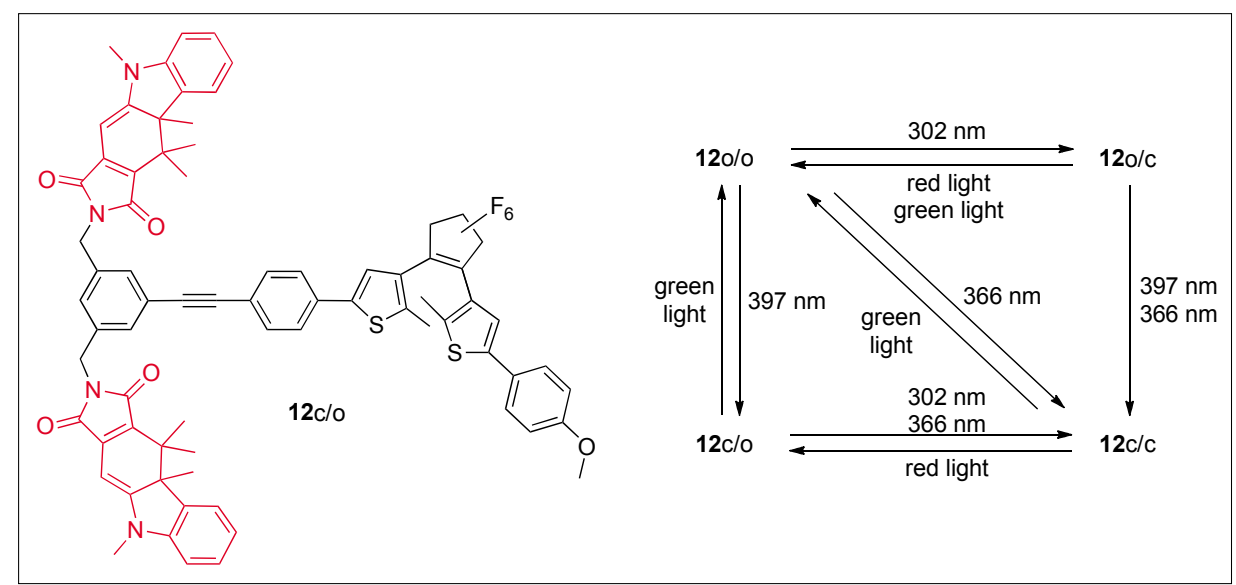

Fig. 10. Structure of the fluorescent 12c/o form of the multiphotochromic platform 12 and network of photochemical transformations of the system.

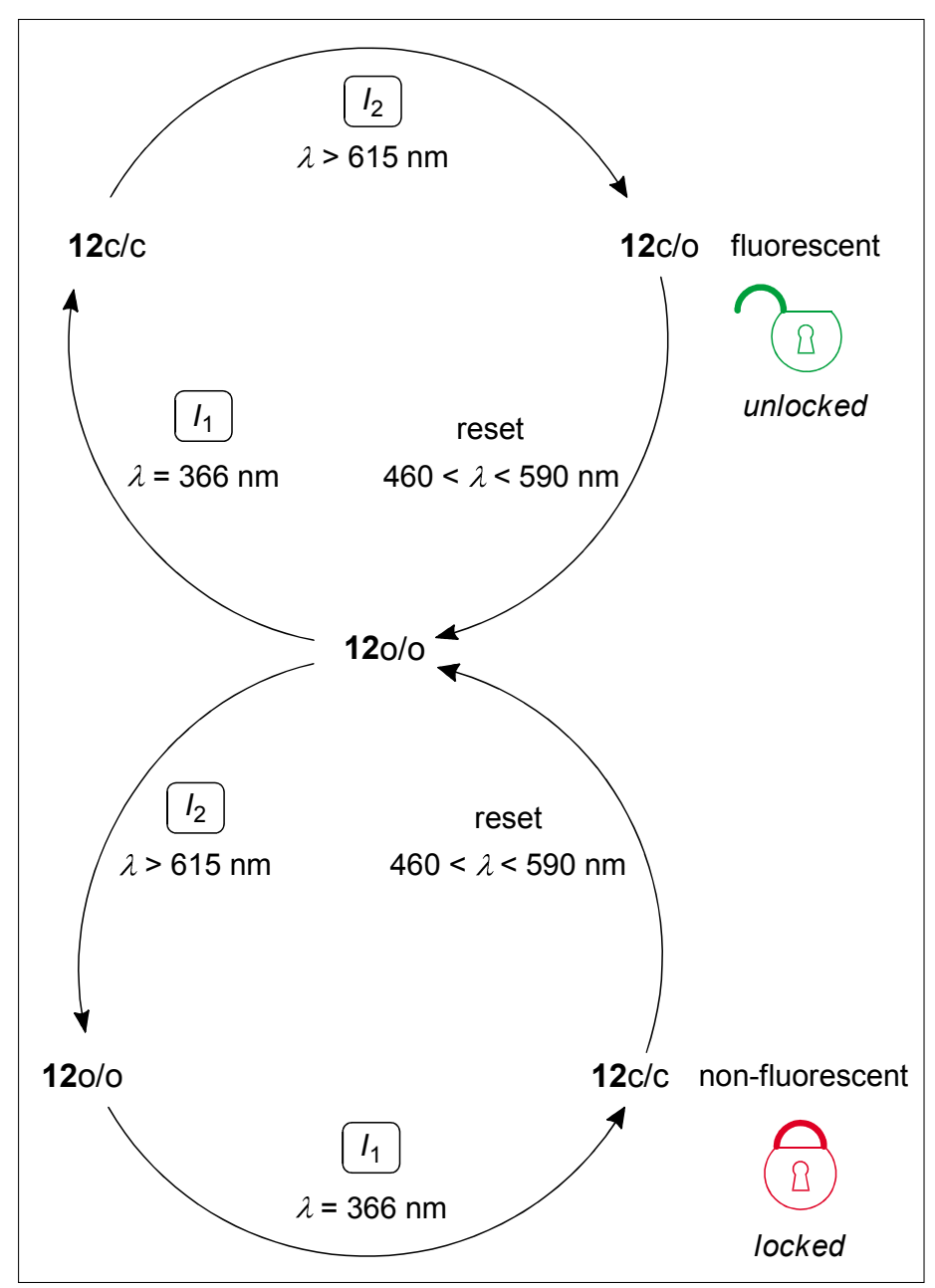

Fig. 11. Molecular keypad function of 12.

tion. Here each of the four binary input combinations $(00,01,10,11)$ is represented by a unique output vector. ${ }^{[52]}$ Due to the manifold possibilities of reading the output channels it is not difficult to find two wavelengths (475 $\mathrm{nm}$ and $625 \mathrm{~nm})$ where each combination of the inputs $(302 \mathrm{~nm}$ for selective dithienylethene ring closing and $397 \mathrm{~nm}$ for selective fulgimide ring closing) results in a different spectral output pattern. In the concrete case the combination of two TRANSFER logic gates leads to the interpretation of a logically reversible operation. This discussion closes the description of our contributions to the implementation of logic functions with molecular photochromic systems. Noteworthy, while in conventional silicon circuitry the described logic functions would require the physical wiring of logic gates, our unimolecular systems are based on functional integration rather than physical integration. Furthermore, they allow the reconfiguration of the operation (changing from one logic function to another) by simply changing excitation conditions or optical 
readout channels. These characteristics are inherent to molecular systems and underpin the advantages of these building blocks for alternative approaches to computing.

\section{Conclusions}

In this account the conceptual approach of using molecules for the realization of simple but also more advanced logic operations was demonstrated. It was shown that on the basis of the universality of logic any type of input signal, such as chemical species or light, can be used to address logic switches and that optical outputs constitute a convenient manner to read the state of the system. In this respect the operation mode of fluorescent chemosensors has a straight relation to molecular logic and information processing. Based on these introductory considerations acid-base switchable fluorescent systems from our own research work were discussed and special emphasis was given to multi-level switches that reach conceptually beyond common binary notations. However, chemical inputs have certain disadvantages that are related to input-output homogeneity and the cumbersome recycling of the switching process. Photochromic switches provide a series of advantages (remote switching, operation without waste products, reversible switching for recycling and resetting, inputs and output of the same physical nature) that identify them as serious candidates for molecular computing. In this account a special focus was put on the use of photochromic molecules for memory-based logic functions. Finally, a very advanced molecular multi-photochromic platform with a so far unsurpassed degree of functional integration was presented.

At the moment two major tendencies in molecular logic can be observed: a) the application as conceptual approach to smart materials and devices for diagnostics and drug delivery (theranostic devices) and b) the development of molecular computers. While it is not hard to imagine that the first trend will lead soon to useful applications, ${ }^{[53]}$ molecular computing in the sense of silicon-based technologies is beyond doubt still a very distant future goal that will require much more outside-the-box thinking involving concerted efforts of chemists, physicists, materials scientists, and engineers. However, as a bottom-up approach it starts with the molecule and the molecular design of functional properties and that is what chemists are supposed to do.

\section{Acknowledgements}

This work has been supported by the Spanish Ministry of Economy and Competitiveness (grants CTQ2008-06777-C02-02 and CTQ2011-28390) and the Andalusian Government (Projects of Excellence P08FQM-3685 and P12-FQM-2140). I am indebted to all co-workers and collaborators that are mentioned in the cited references. I am especially grateful to Joakim Andréasson (Chalmers University of Technology, Göteborg, Sweden) with who we maintain a very fruitful collaboration on photochromic systems since many years. Finally, I would like to express my sincerest gratitude to the Award Committee and the Board of the Swiss Chemical Society for the honor of receiving the GrammaticakisNeumann Award 2013.

Received: June 14, 2014

[1] U. Pischel, B. Heller, New J. Chem. 2008, 32, 395.

[2] A. Aviram, J. Am. Chem. Soc. 1988, 110, 5687.

[3] A. P. de Silva, H. Q. N. Gunaratne, C. P. McCoy, Nature 1993, 364, 42.

[4] A. P. de Silva, 'Molecular Logic-based Computation', The Royal Society of Chemistry, Cambridge, 2013.

[5] J. Andréasson, U. Pischel, Chem. Soc. Rev. 2010, 39, 174.

[6] K. Szaciłowski, Chem. Rev. 2008, 108, 3481.

[7] A. P. de Silva, H. Q. N. Gunaratne, T. Gunnlaugsson, A. J. M. Huxley, C. P. McCoy, J. T. Rademacher, T. E. Rice, Chem. Rev. 1997, 97,1515

[8] A. P. de Silva, S. A. de Silva, J. Chem. Soc., Chem. Commun. 1986, 1709.

[9] A. P. de Silva, R. A. D. D. Rupasinghe, J. Chem. Soc., Chem. Commun. 1985, 1669.

[10] D. C. Magri, G. J. Brown, G. D. McClean, A. P. de Silva, J. Am. Chem. Soc. 2006, 128, 4950.

[11] T. Konry, D. R. Walt, J. Am. Chem. Soc. 2009, $131,13232$.

[12] S. Ozlem, E. U. Akkaya, J. Am. Chem. Soc. 2009, 131, 48 .

[13] S. Erbas-Cakmak, O. A. Bozdemir, Y. Cakmak, E. U. Akkaya, Chem. Sci. 2013, 4, 858.

[14] R. J. Amir, M. Popkov, R. A. Lerner, C. F. Barbas III, D. Shabat, Angew. Chem. Int. Ed. $\mathbf{2 0 0 5}, 44,4378$.

[15] S. Angelos, Y. W. Yang, N. M. Khashab, J. F. Stoddart, J. I. Zink, J. Am. Chem. Soc. 2009, 131,11344

[16] G. E. Moore, Electronics 1965, 38, 114

[17] R. Guliyev, S. Ozturk, Z. Kostereli, E. U. Akkaya, Angew. Chem. Int. Ed. 2011, 50, 9826.

[18] S. Silvi, E. C. Constable, C. E. Housecroft, J. E. Beves, E. L. Dunphy, M. Tomasulo, F. M. Raymo, A. Credi, Chem. Eur. J. 2009, 15, 178.

[19] S. Erbas-Cakmak, E. U. Akkaya, Angew. Chem. Int. Ed. 2013, 52, 11364.

[20] J. Andréasson, U. Pischel, Isr. J. Chem. 2013, 53, 236.

[21] D. Gust, J. Andréasson, U. Pischel, T. A. Moore, A. L. Moore, Chem. Commun. 2012, 48, 1947.

[22] D. Gust, T. A. Moore, A. L. Moore, Chem. Commun. 2006, 1169.

[23] R. Ferreira, P. Remón, U. Pischel, J. Phys. Chem. C 2009, 113,5805

[24] A. P. de Silva, H. Q. N. Gunaratne, J.-L. HabibJiwan, C. P. McCoy, T. E. Rice, J.-P. Soumillion, Angew. Chem. Int. Ed. Engl. 1995, 34, 1728.
[25] J. F. Callan, A. P. de Silva, N. D. McClenaghan, Chem. Commun. 2004, 2048.

[26] G. Dilek, E. U. Akkaya, Tetrahedron Lett. 2000, 41, 3721.

[27] F. Pina, M. J. Melo, M. A. Bernardo, S. V. Luis, E. García-España, J. Photochem. Photobiol. A: Chem. 1999, 126, 65

[28] V. F. Pais, P. Remón, D. Collado, J. Andréasson, E. Pérez-Inestrosa, U. Pischel, Org. Lett. 2011 , 13, 5572.

[29] V. F. Pais, M. Lineros, R. López-Rodríguez, H. S. El-Sheshtawy, R. Fernández, J. M. Lassaletta, A. Ros, U. Pischel, J. Org. Chem. 2013, 78, 7949.

[30] V. F. Pais, H. S. El-Sheshtawy, R. Fernández, J. M. Lassaletta, A. Ros, U. Pischel, Chem. Eur. J. 2013, 19, 6650.

[31] V. F. Pais, J. M. Lassaletta, R. Fernández, H. S El-Sheshtawy, A. Ros, U. Pischel, Chem. Eur. J. 2014, 20, 7638.

[32] C. Di Pietro, G. Guglielmo, S. Campagna, M. Diotti, A. Manfredi, S. Quici, New J. Chem. 1998, 22, 1037.

[33] Z. Tian, A. D. Q. Li, Acc. Chem. Res. 2013, 46, 269.

[34] M. Hammarson, J. Andersson, S. M. Li, P. Lincoln, J. Andréasson, Chem. Commun. 2010, 46,7130 .

[35] Y. Wu, Y. Xie, Q. Zhang, H. Tian, W. Zhu, A. D. Q. Li, Angew. Chem. Int. Ed. 2014, 53, 2090.

[36] M. Berberich, A.-M. Krause, M. Orlandi, F Scandola, F. Würthner, Angew. Chem. Int. Ed. 2008, 47, 6616.

[37] T. Fukaminato, T. Sasaki, T. Kawai, N. Tamai, M. Irie, J. Am. Chem. Soc. 2004, 126, 14843.

[38] T. B. Norsten, N. R. Branda, Adv. Mater. 2001, 13,347

[39] A. Fernández-Acebes, J.-M. Lehn, Chem. Eur J. 1999, 5, 3285 .

[40] Chem. Rev. 2000, 100, 1683-1890. Special Issue on Photochromism: Memories and Switches.

[41] P. Remón, M. Hammarson, S. M. Li, A. Kahnt, U. Pischel, J. Andréasson, Chem. Eur. J. 2011, 17, 6492 .

[42] D. Margulies, C. E. Felder, G. Melman, A Shanzer, J. Am. Chem. Soc. 2007, 129, 347.

[43] U. Pischel, Angew. Chem. Int. Ed. 2010, 49, 1356.

[44] U. Pischel, J. Andréasson, New J. Chem. 2010 34, 2701.

[45] Y. Hirshberg, J. Am. Chem. Soc. 1956, 78, 2304.

[46] G. de Ruiter, M. E. van der Boom, J. Mater Chem. 2011, 21, 17575.

[47] G. de Ruiter, E. Tartakovsky, N. Oded, M. E van der Boom, Angew. Chem. Int. Ed. 2010, 49 , 169

[48] P. Remón, M. Bälter, S. M. Li, J. Andréasson, U. Pischel, J. Am. Chem. Soc. 2011, 133, 20742

[49] J. Andréasson, U. Pischel, S. D. Straight, T. A Moore, A. L. Moore, D. Gust, J. Am. Chem. Soc. 2011, 133, 11641

[50] M. Bälter, S. M. Li, J. R. Nilsson, J. Andréasson, U. Pischel, J. Am. Chem. Soc. 2013, 135, 10230.

[51] J. Andréasson, S. D. Straight, T. A. Moore, A. L. Moore, D. Gust, J. Am. Chem. Soc. 2008, 130 11122 .

[52] P. Remón, R. Ferreira, J.-M. Montenegro, R. Suau, E. Pérez-Inestrosa, U. Pischel, ChemPhysChem 2009, 10, 2004.

[53] U. Pischel, J. Andréasson, D. Gust, V. F. Pais, ChemPhysChem 2013, 14, 28. 